\title{
Errichtung und Betrieb einer Serviceplattform zur Bürgerinformation bei Hochwasser
}

Microtronics Engineering GmbH mit Sitz in Ruprechtshofen hat mit myDatanet ein neues drahtloses Messsystem entwickelt, das in vielen Bereichen einsetzbar ist. Dank drahtloser Messgeräte und Datenübertragung via GPRS sind die gesammelten Daten in Echtzeit jederzeit über Internet verfügbar.

\section{Aufgabenstellung}

Gemeinsam mit den Gemeinden Ruprechtshofen, St. Leonhard/Forst und Zelking/Matzleinsdorf sowie den ansässigen Feuerwehren wurde ein Weg gesucht, das zeit- und kostensparende System myDatanet zum Wohle der Bürger zu nutzen.

Bisher war es für die Feuerwehr notwendig, bei Hochwassergefahr entsprechende Gewässer in kurzen Abständen vor Ort zu kontrollieren, um bei ansteigendem Wasserstand die notwendigen Schutzmaßnahmen wie Alarmierung der Bevölkerung, Aufbau von Schutzdämmen, Sicherung öffentlicher Gebäude etc. rechtzeitig in die Wege zu leiten.

\section{Lösung}

Mit www.wasserstand.info bietet Microtronics den Einsatzkräften der Region Melktal die einfache Möglichkeit, die Pegelstände der Flüsse Mank und Melk über das Internet einzusehen. Die Datenerfassung erfolgt über die digitale Pegellatte, welche vor Ort aus den Pegelsonden besteht, die durch qualifiziertes Fachpersonal in Betrieb gesetzt werden. Mithilfe des Systems myDatanet werden die Messwerte kontinuierlich an das zentrale Datenerfassungssystem geleitet, wobei bei bedrohlichem Pegelstand eine Alarmierung über SMS und E-Mail erfolgt. Somit können im Alarmfall Einsatzkräfte und Anrainer ganz einfach die kritischen Stellen über Internet im Auge behalten. Die zentrale Servereinheit wird von der Firma Microtronics betrieben.

Als Pilotprojekt wurde an der Melkflussbrücke in Ruprechtshofen (Niederösterreich) eine Messstation installiert. Die Möglichkeit, die Messwerte auf www. wasserstand.info und den Seiten der Ge- meinden und Feuerwehren einzusehen, wurde von der Bevölkerung ausgiebig genutzt. Es hat sich gezeigt, dass für eine weitere Optimierung von Einsätzen und die damit erzielbare Schadensminimierung zusätzliche Messstellen erforderlich sind. Nach Maßgabe der örtlichen FF wurden 5 weitere Pegelmessstellen in Zelking/Lunzen, Diesendorf/Hofstetten, Oberndorf/Burmühle und am Zubringerfluss Mank (St. Leonhard/Steghof und Mank/St. Haus) errichtet.

\section{Alarmierung und Serviceplattform}

In Zusammenarbeit mit den Gemeinden und den FFs stellt Microtronics die Bürgerserviceplattform www.wasserstand. info zur Verfügung. Die betroffene Bevölkerung zeigte großes Interesse daran, im Alarmfall möglichst direkt benachrichtigt zu werden. Deshalb wurde das ursprünglich nur für die FF-Mitglieder vorgesehene Alarmierungsservice nun auch für die Bürger freigegeben.

Jeder kann sich zum Erhalt von SMSWarn- und -Alarmnachrichten registrieren. Die Registrierung erfolgt kostenlos, wahlweise auf der Homepage der Gemeinde, Feuerwehr oder über das von Microtronics betriebene Internetportal www. wasserstand.info. Bei Überschreitung der konfigurierten Schwellen werden die örtlichen Feuerwehren alarmiert. Die Feuerwehr analysiert die Lage und löst gegebenenfalls eine Vorwarnstufe oder einen Hochwasseralarm pro Zone aus. Sofort werden alle angemeldeten Bürger per SMS informiert. Nachts, zwischen 22:00 und 7:00 Uhr, wird eine Weckfunktion aktiviert. Diese versendet SMS-Alarmnachrichten automatisch dreifach im Abstand von 3 Minuten.

Über die Alarmierung hinausgehend kann jeder, auch nicht registrierte Benutzer, aktuelle Pegelstände sowie Trendlinien über beliebige Zeiträume abrufen.

Im Normalfall (kein Hochwasser, grüne Markierung) erfolgt die Messung alle 2 Minuten und das Internetportal wird stündlich aktualisiert. Ab Überschreitung der Voralarm-Stufe (orange Markierung) erfolgt die Aktualisierung am Internetportal alle 10 Minuten. Das Überschreiten der Voralarm- bzw. Alarmgrenzen wird, unabhängig vom Aktualisierungsintervall, immer unmittelbar signalisiert. Im Alarmfall werden betroffene Zonen rot dargestellt.

\section{Außenstationen}

Es kommen autarke, batterie-, akku- oder solarbetriebene Erfassungsgeräte zum Einsatz. Daher ist grundsätzlich kein Stromanschluss an der Messstelle erforderlich, was den Einbau der Messsonden an jeder erdenklichen Stelle der Gewässer erlaubt. Um regelmäßiges Wechseln der Batterien zu vermeiden, kann auch auf netzbetriebene Erfassungsgeräte umgeschwenkt werden, sofern ein Stromanschluss gegeben sein sollte. Im konkreten Fall wurden akkubetriebene myDatalogEASY-Messgeräte verwendet.

Neben dem Pegelstand werden auch Betriebssignale und GSM-Feldstärke aufgezeichnet. Durch die integrierte Systemalarmierung werden bei Ausfall des Gerätes oder leerem Akku Alarmmeldungen per E-Mail oder SMS übertragen. Somit ist die Sicherheit gegeben, über Störungen einzelner Messstationen rund um die Uhr informiert zu sein.

\section{Übertragung der Daten}

Die Daten werden in den Messgeräten zwischengespeichert (bis zu 50.000 Messwerte) und mit einem speziellen Protokoll beim nächsten Übertragungsintervall über das Internet an die Zentrale übermittelt. Mess- und Übertragungsintervalle sind ab 1 Minute unabhängig von einander frei konfigurierbar. Für die Datenübertragung ist eine SIM-Karte (GPRS-Datenkarte) notwendig. Dank des extrem schlanken Protokolls kommt myDatanet mit geringem Datenvolumen aus.

\section{myDataSIM}

myDataSIM wurde bereits fertig konfiguriert in das Messgerät eingebaut, sodass das Gerät nur noch eingeschaltet werden muss und die Messung beginnen kann. 


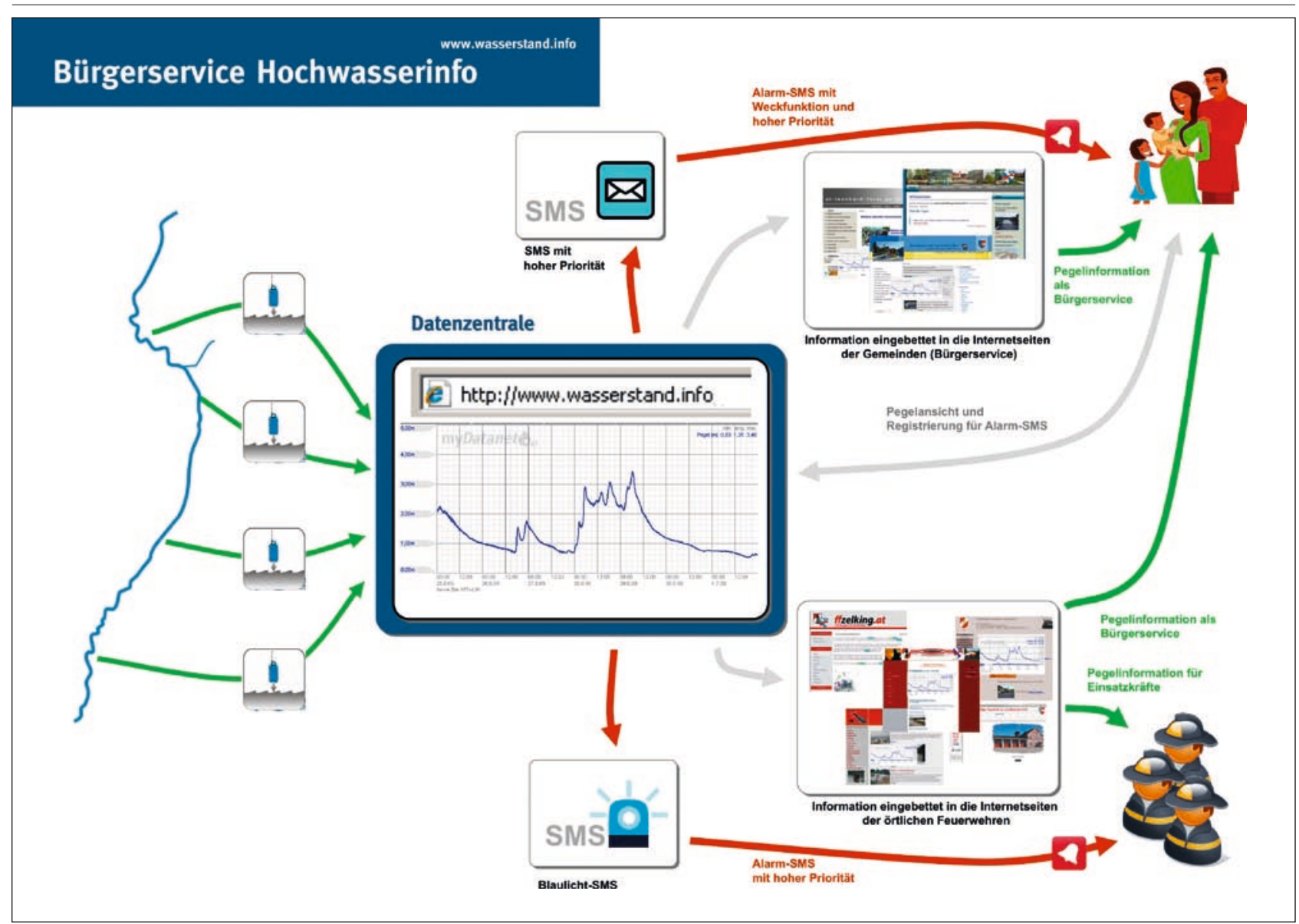

Abb. 1: Funktionsweise.

Ein weiterer Vorteil der myDataSIM-Karte ist die automatische Auswahl des Netzproviders. So kann die Karte je nach Feldstärke der einzelnen Provider zwischen A1, T-Mobile und Orange automatisch wechseln. Das erhöht die Verbindungssicherheit wesentlich und gewährleistet eine sorgenfreie Kommunikation. Funknetzeinrichtungen oder Ähnliches sind nicht erforderlich.

\section{Daten Client}

Für die professionelle Nutzung der Daten kann der Download mit einem Zusatzprogramm von Microtronics automatisiert werden. So können TSV-Files im Tages-, Wochen- oder Monatsintervall automatisch in vordefinierbaren Ordnern auf einem lokalen Rechner abgespeichert werden. Diese Daten sind Excel-kompatibel und können auch mit anderen Tabellenkalkulationsprogrammen weiterverarbeitet werden. Dieses Zusatzprogramm kann auf mehreren Rechnern installiert werden, damit können einzelne Bearbeiter die für sie wichtigen Informationen automatisch aus dem System beziehen.
Der automatisierte Datenaustausch mit weiterführenden Systemen kann über XML vollautomatisiert erfolgen, was z. B. für den Zugriff des Hydrografischen Dienstes sinnvoll ist.

\section{Vorteile}

- Durch die frühzeitige Warnung der Bevölkerung kann diese bereits vor Eintreten eines Ernstfalls Vorbereitungen treffen, wie z. B. Keller räumen, Kraftfahrzeuge aus dem Gefahrenbereich bringen, Türen und eventuell Fenster abdichten, Sandsäcke schlichten und je nach Ernst der Lage gegebenenfalls sich selbst und Angehörige in Sicherheit bringen.

- Durch den Wegfall des manuellen Ablesens der Pegellatte an den einzelnen Flussabschnitten können die Einsatzzeiten drastisch minimiert werden, was für die zahlreichen ehrenamtlichen Mitarbeiter v. a. nachts eine wesentliche Erleichterung ist.

- Durch die Platzierung mehrerer Messstellen im Flusslauf und im Zubringer ist auch bei unvorhergesehenem, ra- schem Ansteigen des Wasserstandes eine frühzeitige Alarmierung gewährleistet.

- Durch die integrierte Systemalarmierung wird der Ausfall eines Messinstrumentes sofort gemeldet, sodass eine lückenlose Erfassung des Pegelstandes dennoch möglich ist.

- Das System von myDatanet macht es außerdem möglich, dass Geräte, die entnommen werden, problemlos und ohne Verlust von Daten oder Einstellungen ersetzt werden können. Dafür sind nur ein paar Mausklicks notwendig.

- Bei Bedarf kann das System ohne großen Aufwand erweitert werden.

- Anwender des Systems schätzen vor allem die einfache Bedienung und die übersichtlich dargestellten Informationen in Echtzeit. Die schnelle und bequeme Verfügbarkeit der Information ermöglicht einen raschen und der Situation angemessenen Einsatz des Teams.

„Die Hochwasserereignisse des Sommers 2009 haben deutlich gezeigt, dass das bestehende System sehr hohen Nutzwert für 
die Freiwillige Feuerwehr als auch für die Bevölkerung hat. So konnten vor allem die nächtliche Vor-Ort Präsenz minimiert und Einsatzzeiten sowie Folgeschäden reduziert werden. Über 2500 Internet-Zugriffe in 7 Tagen geben ein deutliches Bild von der bereits guten Akzeptanz!", freut sich Alfred Riedl, Feuerwehrzugskommandant in Ruprechtshofen über den Erfolg der produktiven Zusammenarbeit zwischen Microtronics, Freiwilliger Feuerwehr und Gemeinden.

Die Bürgermeister der am Projekt beteiligten Gemeinden sind sich einig: „Bei hohem Pegelstand muss unsere Feuerwehr nun nicht mehr rund um die Uhr den Fluss vor Ort im Auge behalten und auch unsere Bevölkerung kann ruhiger schlafen - eine eindeutige Steigerung der Lebensqualität.“

„myDatanet kann zwar kein Hochwasser verhindern, aber durch die Information über den aktuellen Pegelstand helfen die negativen Auswirkungen abzuschwächen“, freut sich Hans Peter Buber, Geschäftsführer von Microtronics, über das gelungene Projekt.

\section{Weitere Einsatzmöglichkeiten von myDatanet}

Neben dem Einsatz für die Kontrolle des Wasserstands, wie sie derzeit z. B. als digitale Pegellatte im Melktal getestet wird, kann dieses System natürlich noch für eine Reihe anderer Anwendungen sinnvoll sein: Derzeit wird das Messsystem häufig in der Kanalisation zur Niveau- und Durchflussüberwachung oder aber auch zum Gasmonitoring in Kanalschächten eingesetzt, wie z. B. in Wiener Neustadt, Brüssel, Kopenhagen oder Berlin. Durch Kombination der Messergebnisse mit zusätzlichen Informationen von Wetterstationen oder über Niederschläge haben Kanalbetreiber den bestmöglichen Überblick über die Geschehnisse in ihrem Kanal.

Kombiniert man die oben erwähnten Module mit drahtlosen, akkubetriebenen, ATEX-zertifizierten Messgeräten, bieten sich nahezu unbegrenzte Anwendungsmöglichkeiten:

- Gasmonitoring (in ATEX-Zonen, zur Korrosions- und Geruchsbekämpfung),

- Niveau- und Durchflussüberwachung (Verbrauchsmengenerfassung, Tagesmengenerfassung, Grundwasserpegelüberwachung, Hochwasserfrüherkennung etc.),

- Füllstandsüberwachung (Verbrauchsüberwachung, bei Silo- und Tankanla-

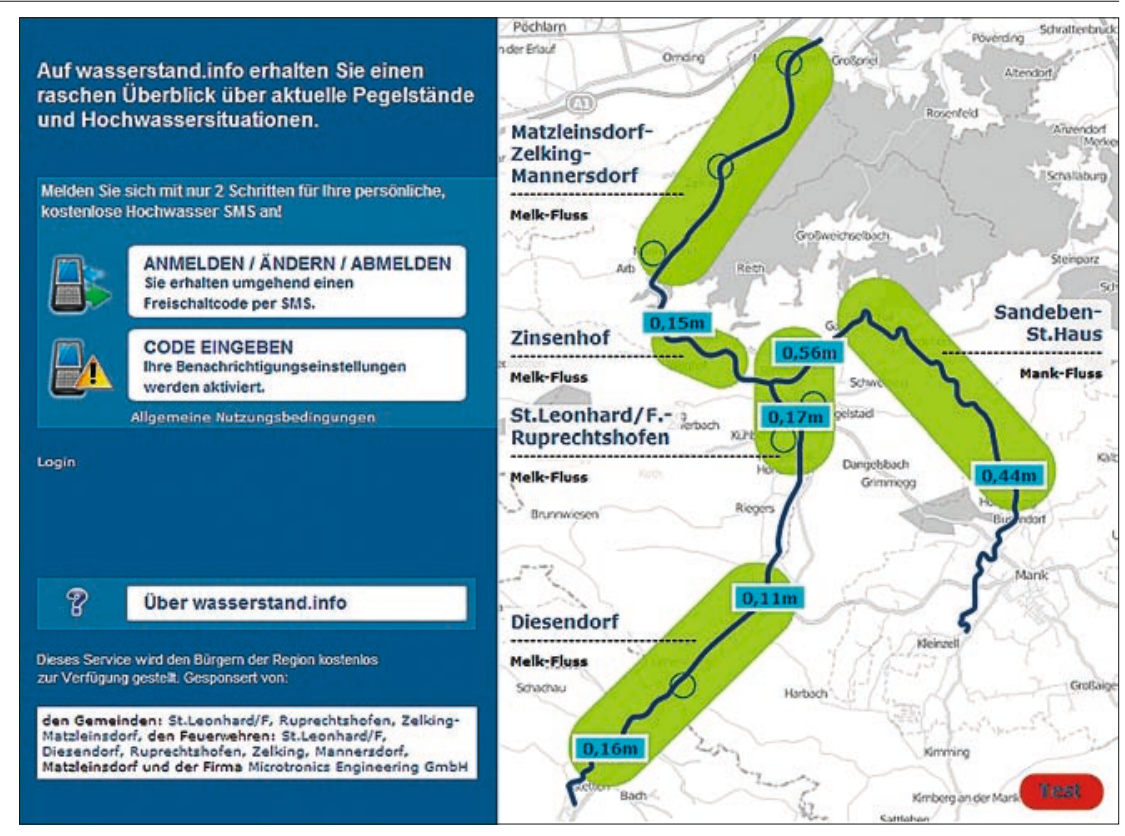

Abb. 2: Startseite der Serviceplattform.

\section{Zonenübersicht > Melk / Ruprechtshofen}
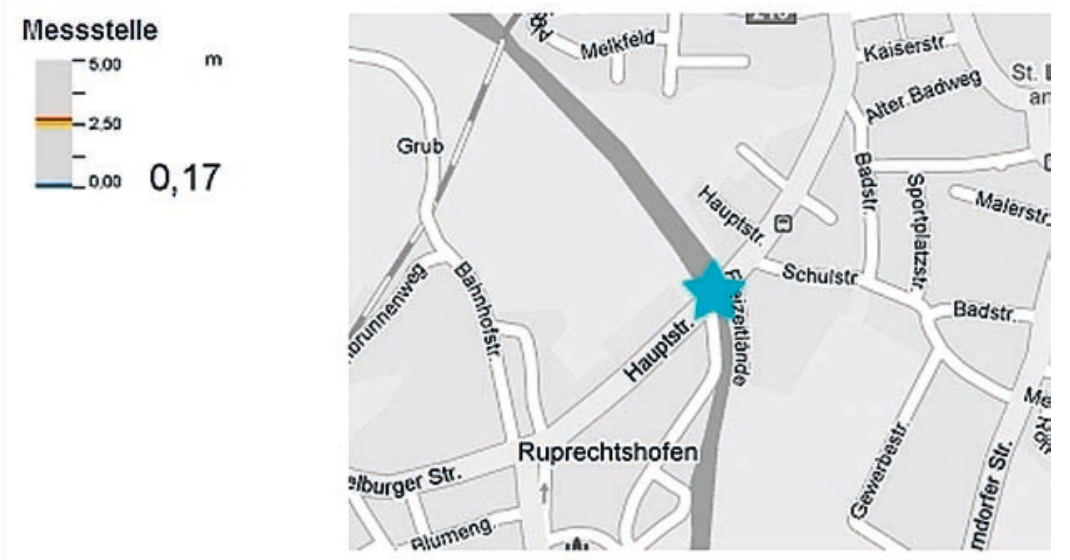

\begin{tabular}{lll}
$4 h$ & $4 \mathrm{~h}$ & Woche \\
\hline
\end{tabular}

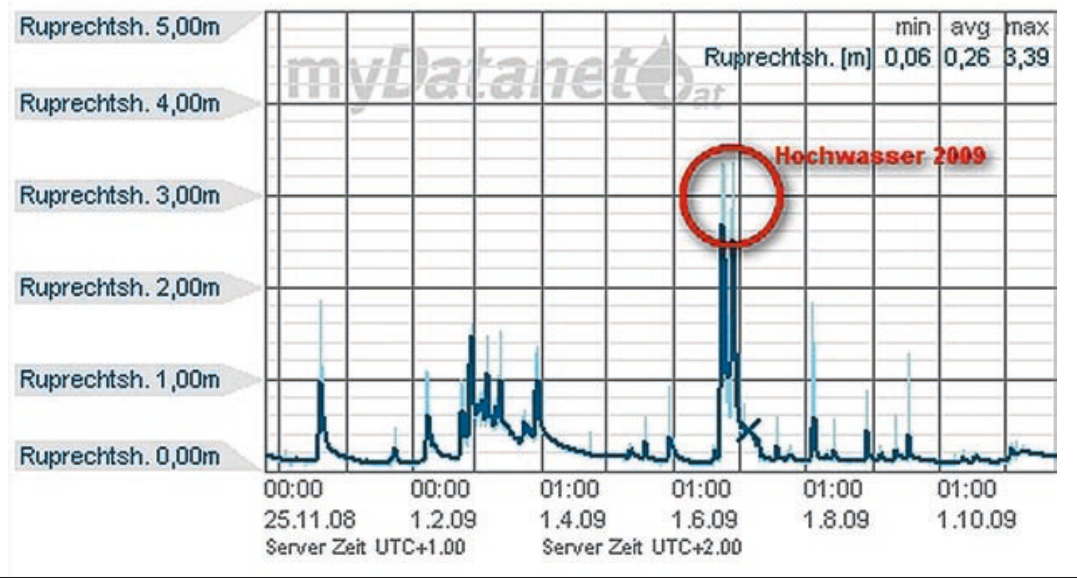

Abb. 3: Messwerte. 
gen mit direkter Anbindung an das Lieferantensystem etc.),

- Zählerfernauslesung (z.B. automatisierte Gas-, Wasser-, Stromzählerauslesung)

- Verkehr (Ozon- und Lärmmessung mit Darstellung auf Karten, Niederschlag, Temperatur, Wind, Ansteuerung für automatische Verkehrsbeeinflussungsanlagen etc.),

- Emissionen (Ausbreitungsberechnungen, ATEX-Zonen etc.),

- Raumklima (für Museen, Lagerhallen, Produktionsstätten etc.),

- Wetter (für Kommunal-, Agrar- und Industrieanlagen),

- Pumpen (automatische Pumpenregelung, Dosierung etc.),

- Logistik (Füllstand und Position von Containern, Silo etc.),

- Pipeline (Gas-, Füllstand-, Durchflussund Drucküberwachung).

\section{Zusammenfassung}

In Zusammenarbeit mit Gemeinden aus dem Melktal sowie deren Freiwilligen Feuerwehren hat Microtronics Engineering $\mathrm{GmbH}$ eine Möglichkeit gesucht, das von ihr entwickelte, neuartige Messsystem my-

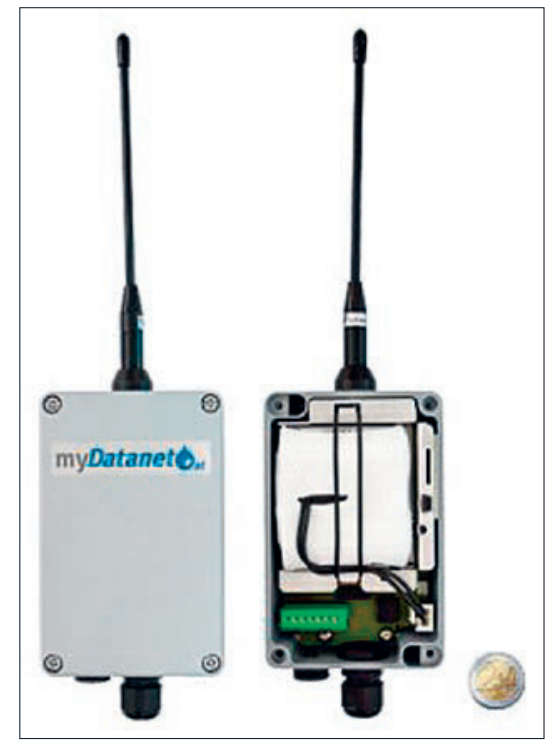

Abb. 4: myDatalogEASY.

Datanet zum Wohle der Bevölkerung zu nutzen und „digitale Pegellatten“ installiert. Drahtlose, akkubetriebene Messgeräte erheben an insgesamt 6 Messpunkten im Melkfluss und dem Zubringerfluss Mank Pegelstände. Mithilfe des myDatanet Systems werden die erfassten Messwerte drahtlos, mittels GPRS an den Server ge- sendet. Sie sind mittels myDataweb über Internet abrufbar. Für Einsatzkräfte, Anrainer und Interessierte hat Microtronics die Serviceplattform www.wasserstand.info eingerichtet. Dort kann jedermann jederzeit die aktuellen Pegelstände in Echtzeit einsehen. Es besteht auch die Möglichkeit, sich zu registrieren, um im Warn- und Alarmfall per SMS informiert zu werden. Die Warn- und Alarmwerte werden von den örtlichen Feuerwehren verwaltet.

Dank der Serviceplattform, die in Echtzeit verfügbare Daten und eine übersichtliche grafische Aufbereitung bietet, sind die Betroffenen über das Geschehen an der entsprechenden Messstelle sofort im Bilde und können dementsprechend reagieren.

Der Einsatz dieses Systems hat sich lt. den Verantwortlichen bei den Gemeinden und örtlichen Freiwilligen Feuerwehren schon nach kurzer Zeit bewährt und wird auch von der Bevölkerung bereits intensiv genutzt.

\section{Informationen:}

Microtronics Engineering $\mathrm{GmbH}$

Hauptstraße 7

3244 Ruprechtshofen

Tel.: +43-2756-7718

E-Mail: office@microtronics.at

Internet: www.microtronics.at, www.mydatanet.at 